\title{
SIMULAÇÃO NUMÉRICA DO SOPRO DE OXIGÊNIO ATRAVÉS DE BOCAL SUPERSÔNICO
}

\author{
Breno Totti Maia ${ }^{\prime}$ \\ José Eduardo Mautone Barros ${ }^{2}$ \\ Leandro Miranda Nascimento ${ }^{3}$ \\ Marcelo de Souza Lima Guerra ${ }^{4}$ \\ Roberto Parreiras Tavares ${ }^{5}$
}

\section{Resumo}

Neste trabalho é desenvolvida uma análise do escoamento de oxigênio em bocais de lança supersônica via CFD e são elaboradas propostas de alterações visando melhorar o seu desempenho. A metodologia envolve o uso de CFD para simular os campos de velocidade, pressão, massa específica e temperatura do oxigênio. Avalia-se o comportamento do oxigênio ao longo do bocal, desde a região de elevada pressão e baixa velocidade na entrada, até a condição supersônica na saída. Os valores obtidos são comparados com resultados analíticos para condições ideais e são propostas alterações na geometria para reduzir prováveis perdas de carga. Em seguida, são avaliados o comportamento do jato e sua interação com o ambiente. São analisadas propostas de novas geometrias, com o objetivo de maximizar a velocidade do jato.

Palavras-chave: Escoamento supersônico; CFD; Pressão; Gás.

\section{NUMERICAL SIMULATION OF BLOW THROUGH SUPERSONIC NOZZLES}

\begin{abstract}
In the present work, the behavior of oxygen flow in supersonic nozzles lance is analyzed by CFD and design changes to improve performance are proposed. The methodology involves the use of CFD in the design of the tip nozzle. The behavior of oxygen jet along the nozzle is analyzed, since the region of high pressure and low speed until the entrance to the supersonic condition at the output. The values obtained are compared to analytical results for ideal conditions and geometry changes to reduce losses are proposed. The behavior of the jet and its interaction with the environment are also investigated. The new geometries proposed are analyzed in order to maximize the jet speed.
\end{abstract}

Key words: Supersonic flow; CFD; Pressure; Gas.

\section{INTRODUÇÃO}

Convertedores BOF têm como objetivo principal a remoção de carbono do ferro-gusa para a sua transformação em aço. Esse processo dá-se a partir da reação do carbono dissolvido no ferro gusa com o oxigênio, formando $\mathrm{CO}_{(\mathrm{g})}$ e $\mathrm{CO}_{2(\mathrm{~g})}$ e diminuindo a concentração de carbono no banho metálico.

Para tal, são empregadas atualmente lanças para sopro de oxigênio atingindo velocidades supersônicas, alcançadas devido às elevadas pressões no reservatório e por um correto dimensionamento do bocal, contribuindo para a penetração do oxigênio no banho metálico de alta massa específica.

Atualmente, softwares de fluidodinâmica computacional (CFD), por meio de técnicas de elementos ou volumes finitos, conseguem obter, não apenas as médias das grandezas, como as equações de Glass e Smith, ${ }^{(1)}$ mas também valores pontuais no domínio analisado de soluções. Neste trabalho é desenvolvida uma análise do compor-

\footnotetext{
'Engenheiro Metalurgista, Pesquisador da Lumar Metals, Rod. MG 232, Km 09, 100, Cep 35I62-970, Santana do Paraíso, MG, Brasil. Email:breno.totti@lumarmetals.com.br

${ }^{2}$ Professor PhD., Departamento de Engenharia Mecânica - DEMEC, Escola de Engenharia, Universidade Federal de Minas Gerais - UFMG,

Av. Antônio Carlos, 6627, Pampulha, Cep 31270-90I, Belo Horizonte, MG, Brasil. Email: mautone@demec.ufmg.br

${ }^{3}$ Engenheiro Metalurgista da Gerdau Cearense, Av. Parque Oeste, I400, Distrito Industrial, Cep 61939-I20, Fortaleza, CE, Brasil.

Email: leandromn.metalurgica@yahoo.com.br

${ }^{4}$ Engenheiro Mecânico e Pesquisador da Lumar Metals, Rod. MG 232, Km 09, 100, Cep 35I62-970, Santana do Paraíso, MG, Brasil.

Email: marcelo.guerra@lumarmetals.com.br

${ }^{5}$ Professor PhD., Departamento de Engenharia Metalúrgica e de Materiais - DEMET, Escola de Engenharia, Universidade Federal de Minas Gerais UFMG, Av. Antônio Carlos, 6627, Pampulha, Cep 31270-90I, Belo Horizonte, MG, Brasil. Email: rtavares@demet.ufmg.br
} 
tamento de bocais de lança supersônica via CFD e são propostas alterações visando melhorar o seu desempenho.

$O$ presente trabalho tem como objetivo utilizar o software ANSYS CFX para estudar o comportamento do jato de oxigênio no bocal da lança e estabelecer uma comparação com modelo teórico analítico aplicado a bocais usados rotineiramente em convertedores LD. Este trabalho visa, também, aplicar simulações via ANSYS CFX para comparar o seu desempenho quanto à turbulência $e$ perturbações internas aos bocais e a interação do jato com a atmosfera, propondo novas geometrias para o bocal.

\section{REVISÃO BIBLIOGRÁFICA}

Os conceitos envolvidos em jatos sônicos têm origem nas equações de conservação de energia, conservação de massa e equações de estado aplicadas em sistemas no estado estacionário. Para simplificar os cálculos desenvolvidos, o oxigênio é considerado como gás ideal. $A$ equação de conservação de energia permite estabelecer uma relação entre a velocidade do fluido, a mudança de energia potencial, a energia associada ao nível de pressão e o trabalho feito pelo fluido.

Uma primeira simplificação da equação de Bernoulli, para descrever o comportamento de jatos sônicos, consiste em considerar um tubo na horizontal, considerar regime turbulento, desprezando os efeitos das forças de atrito e, finalmente, considerar que nenhum trabalho nas vizinhanças é realizado. Além dessas simplificações, para fluidos compressíveis, a equação é diferenciada em termos da velocidade média, considerando o escoamento unidimensional. Desta equação ficam estabelecidas relações entre a velocidade, pressão e massa específica, que necessitam da introdução de outras relações para compor um sistema de equações.

Shapiro e Moran ${ }^{(2)}$ descrevem a onda de som como uma onda de pressão que provoca uma pequena perturbação que se propaga através de um gás, líquido ou sólido, com uma velocidade " $v_{s}$ ", e que depende das propriedades do meio. Assim, aplicando o princípio de conservação de massa para perturbação provocada por essa onda sonora, é possível estabelecer uma relação entre a velocidade e a massa específica. Por meio de um balanço forças, ainda nesse ambiente, fica estabelecida uma relação entre a variação da velocidade com a variação da pressão. Considerando a variação na seção transversal do duto, por relações previamente estabelecidas, obtém-se a Equação I:

$$
\frac{d A}{A}=-\frac{d v}{\bar{v}} \times\left(I-M a^{2}\right)
$$

onde: "Ma" - número adimensional de Mach, "A" - área e "v" - velocidade.

O número adimensional de Mach representa uma relação entre a velocidade do fluido e a velocidade do som no meio. A Figura I apresenta a evolução da velocidade de subsônica a supersônica em relação à área.

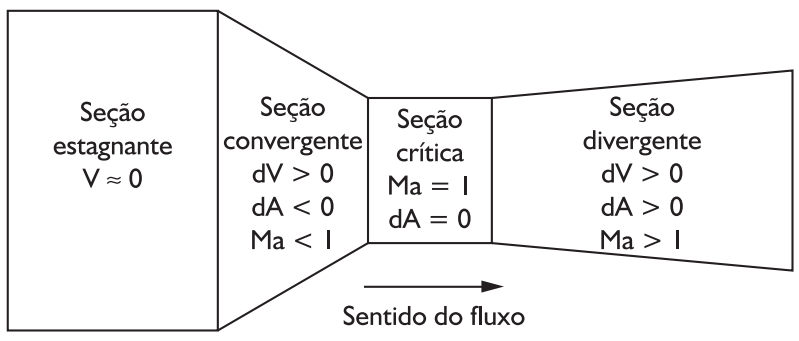

Figura I. Entrada e saída de um bocal supersônico.(3)

O desenvolvimento das expressões que relacionam pressão, temperatura e massa específica do gás é trabaIhoso e considera um balanço de energia em um processo adiabático e isentrópico, fornecendo relações entre dois pontos quaisquer ao longo do duto, representadas pelas Equações 2 a 4:

$$
\begin{aligned}
& \mathrm{T}_{1} \cdot\left(I+\left(\frac{\gamma-1}{2}\right) \cdot \mathrm{Ma}_{1}{ }^{2}\right)=\mathrm{T}_{2} \cdot\left(I+\left(\frac{\gamma-1}{2}\right) \cdot \mathrm{Ma}_{2}{ }^{2}\right) \\
& \mathrm{P}_{1}^{(\gamma-1) / \gamma} \cdot\left(I+\left(\frac{\gamma-1}{2}\right) \cdot M \mathrm{Ma}_{1}^{2}\right)= \\
& \mathrm{P}_{2}^{(\gamma-1) / \gamma} \cdot\left(I+\left(\frac{\gamma-1}{2}\right) \cdot \mathrm{Ma}_{2}{ }^{2}\right) \\
& \rho_{1}^{\gamma-1} \cdot\left(I+\left(\frac{\gamma-1}{2}\right) \cdot \mathrm{Ma}_{1}{ }^{2}\right)= \\
& \rho_{2}^{\gamma-1} \cdot\left(I+\left(\frac{\gamma-1}{2}\right) \cdot \mathrm{Ma}_{2}{ }^{2}\right)
\end{aligned}
$$

onde: "T" - temperatura, "P" - pressão, “ $\rho$ " - massa específica, " $\gamma$ ” - constante adiabática dos gases, "Ma" - número adimensional de Mach. Os subscritos I e 2 representam dois pontos quaisquer do duto.

As equações de temperatura, pressão e massa específica, expressos pelas Equações 2 a 4 representam valores médios ao longo do eixo do comprimento do jato, determinado pela Equação 5, não sendo possível avaliar as variações de propriedades na seção do bocal.

$$
\frac{A}{A_{*}}=\left(\frac{\left(I+\left(\frac{\gamma-1}{2}\right) \cdot M a^{2}\right)}{\left(\frac{\gamma+1}{2}\right)}\right)^{+(\gamma+1) / 2 \cdot(\gamma-1)} \cdot \frac{1}{M a}
$$

onde: "A" - área, "A*" - área da garganta, " $\gamma$ " - constante adiabática dos gases, "Ma" - número adimensional de Mach. 
As mudanças nas propriedades do fluxo são graduais em todos os casos, exceto quando a relação $A / A^{*}$ está próxima da unidade, onde as características do fluxo são alteradas rapidamente com pequenas alterações na seção. Essa região é chamada de transônica, pois ocorre a passagem do regime subsônico para o regime supersônico.

As variações das propriedades do gás podem ser expressas na forma gráfica em função do número de Mach, conforme apresentado na Figura 2.

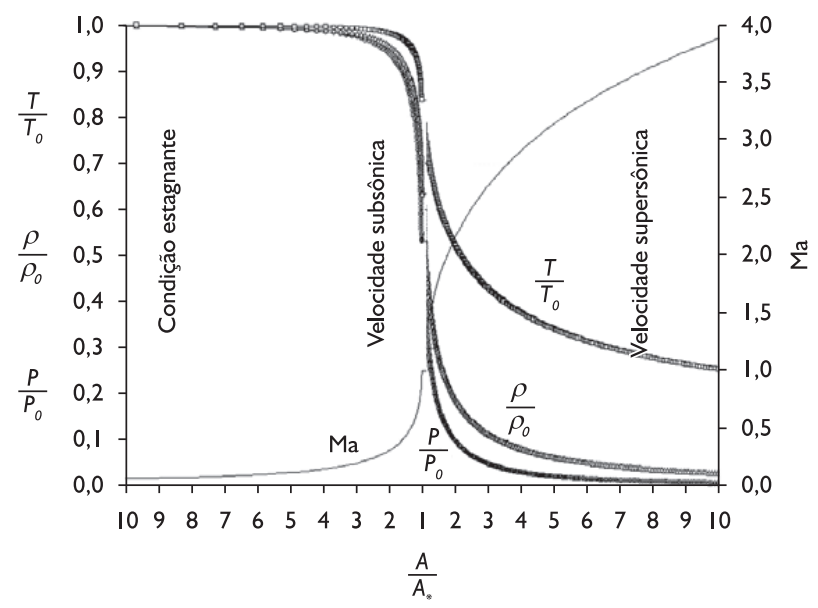

Figura 2. Variação das propriedades do fluxo com a variação $A / A^{*}$.(I)

Para obter informações das propriedades em uma determinada seção faz-se uso de modelos matemáticos. O software CFD consiste em uma ferramenta computacional que resolve sistemas de equações a partir de condições de contorno do problema pela discretização do domínio em pequenos elementos de volume. Balanços de massa, movimento e calor são resolvidos para cada elemento. Os erros no fechamento dos balanços são conhecidos como resíduos, e que devem ser minimizados durante a execução da simulação.

Um recurso dessa ferramenta - os modelos de turbulência - busca descrever escoamento de um fluido em regime turbulento, contornando o problema das elevadas flutuações de velocidade.

\section{MATERIAIS E MÉTODOS}

No desenvolvimento do trabalho foi utilizado - software comercial ANSYS CFX-12 para simulação numérica. $O$ resíduo especificado é de $10^{-6}$, ou seja, o erro no fechamento das equações de conservação deve ser inferior a $0,0001 \%$. O ANSYS CFX-12 calcula os valores das características do escoamento nos pontos nodais, sendo necessária a avaliação dessas características nas interfaces entre os volumes de controle. Para realizar esta interpolação, foi utilizado o esquema High Resolution, que emprega um método mais acurado de interpolação, envolvendo mais pontos nodais. Esse método é menos robusto, ○ que gera dificuldades de convergência da técnica numérica. Para contornar essa dificuldade, foram obtidas soluções iniciais com o esquema Upwind, que é mais robusto, mas menos acurado. A solução obtida por este esquema foi utilizada como ponto de partida para $\circ$ esquema High Resolution.

Nas simulações foram consideradas as propriedades do oxigênio puro, admitido como gás ideal e uma condutividade térmica de $0,02674 \mathrm{~W} /(\mathrm{m} . \mathrm{K})$. Foram realizados dois grupos de simulações: no primeiro foi considerado somente - Bocal para avaliar o aparecimento de ondas de choque em função da parede, representado na Figura 3 pela região $A$. Já no segundo grupo de simulações foi ampliado o domínio, incluindo a região $B$ mostrada na Figura 3 , que corresponde à expansão do jato supersônico para o meio. Desta forma, pode-se avaliar os efeitos da parede, e o comportamento do jato em função da geometria do bocal.

O modelo de turbulência utilizado é o SST (Shear Stress Transport Model), que utiliza o modelo " $\kappa-\omega$ " de turbulência e frequência para regiões de parede e " $\kappa-\varepsilon$ " para centro do fluxo.(4) Esta combinação de funções garante uma transição suave entre os dois modelos e regime de deslocamento do fluido. Em comparação com o tradicional modelo de turbulência " $\kappa-\varepsilon$ ", as geometrias de bocais são apresentadas na Figura 4, em função do diâmetro crítico.

Em todos os casos, o diâmetro de entrada, crítico e o diâmetro de saída permanecem os mesmos, assim como o comprimento total, alterando-se apenas o perfil do bocal.

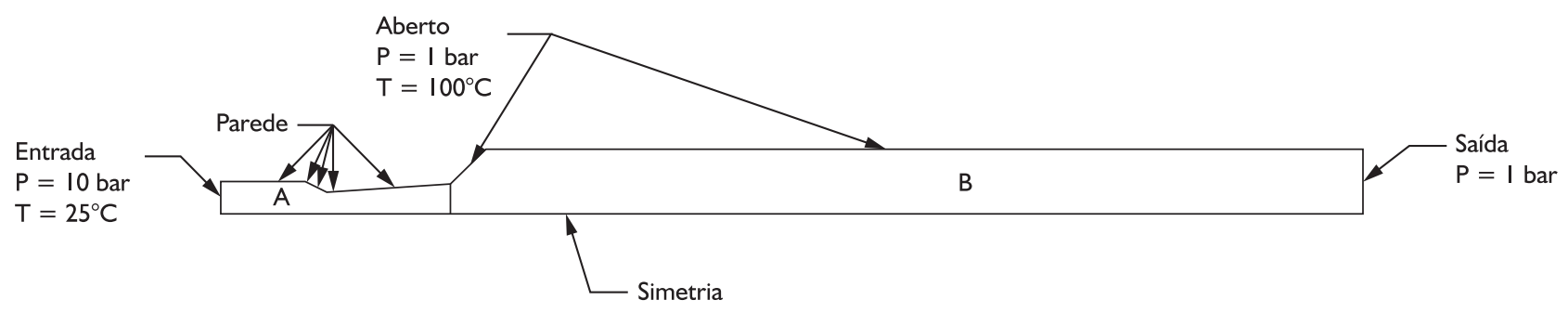

Figura 3. Condições de contorno utilizadas nas simulações. 

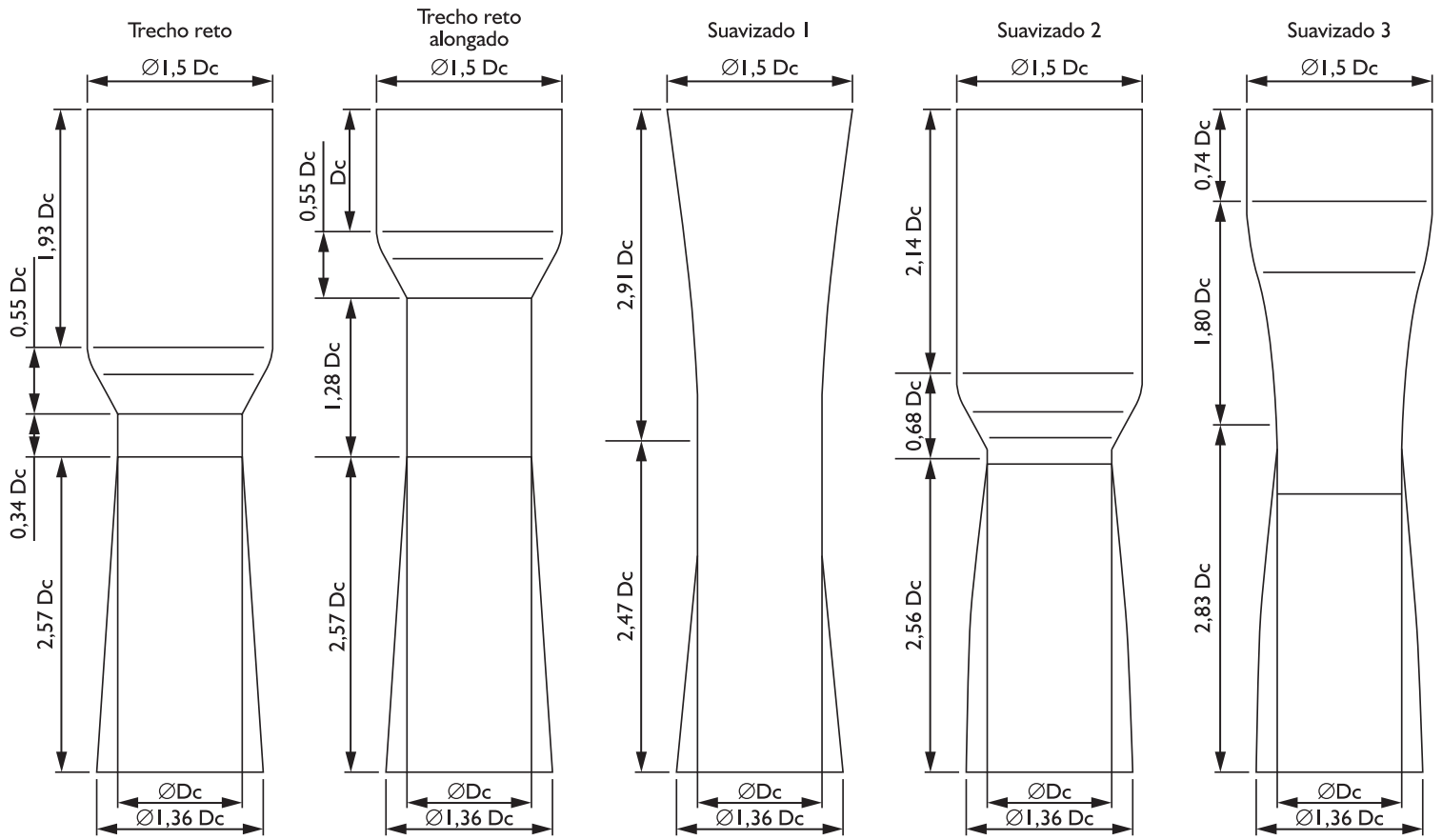

Figura 4. Geometria dos bocais simulados.

\section{RESULTADOS E DISCUSSÃO}

Na primeira simulação foi considerada uma pressão de 10 bar e os parâmetros de um bocal tradicional da indústria siderúrgica com a presença do Trecho Reto Alongado na região da garganta, apresentada na Figura 5.

Comparando as Figuras 2 e 5, é possível notar uma perturbação no comportamento da pressão, massa específica e temperatura. Essa perturbação na região transônica está associada à presença do trecho reto. Como em todo comprimento do trecho reto, a relação de área é igual à unidade, e as variações de propriedades não são claras. Entretanto, por simulação numérica é possível constatar o comportamento da velocidade, conforme apresentado na Figura 6.

$\mathrm{Na}$ Figura 6, a escala é restrita na região da velocidade sônica, ou $M a=I$. É possível notar que existem expansões de ondas limitadas pelo trecho reto, gerando regiões de baixa velocidade dentro do comportamento sônico. Portanto, o trecho reto não respeita a correta relação de área, perturbando a transição das propriedades do oxigênio, quando comparado com a curva ideal.

Foram realizadas simulações reduzindo o comprimento do trecho do reto a um pequeno elemento de área. O comprimento da seção divergente foi aumentado para manter o comprimento total do bocal. O resultado desta simulação é apresentado na Figura 7, para o bocal suavizado I.

Os resultados da Figura 7 são próximos da curva analítica, com pequenas diferenças de valores. Em comparação com a Figura 2, os resultados permitem concluir

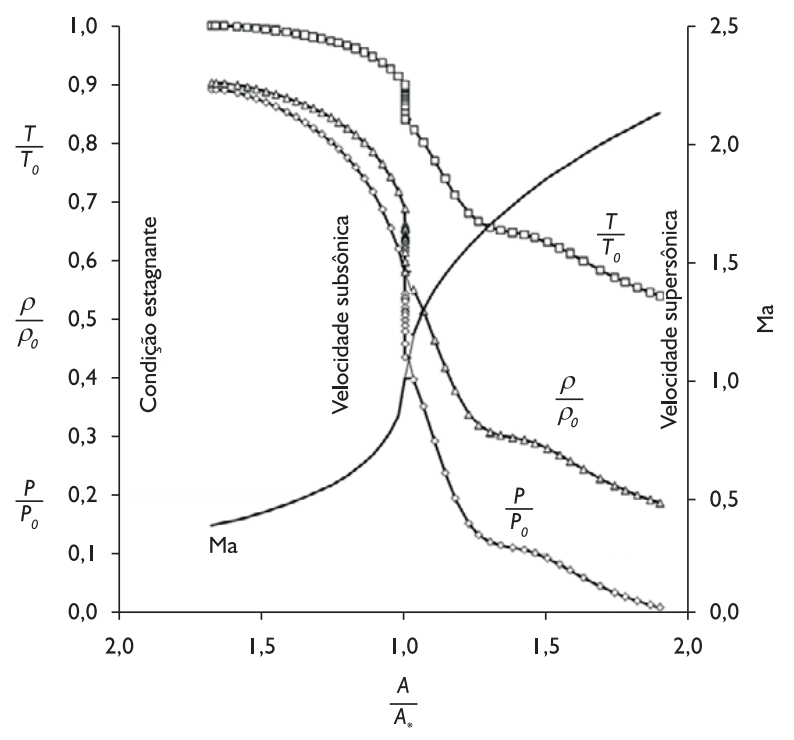

Figura 5. Variação das propriedades do fluxo com a variação $A / A^{*}$ para o Bocal com Trecho Reto.

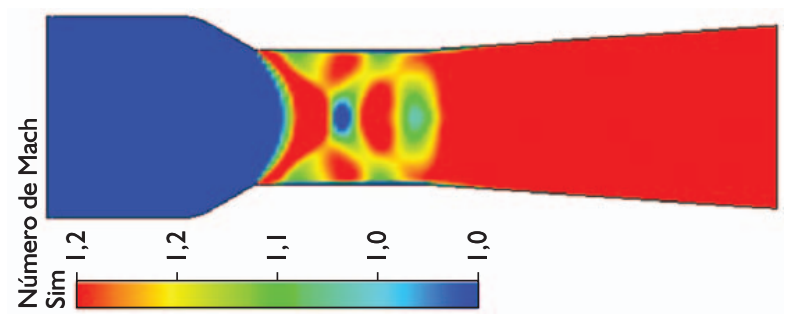

Figura 6. Avaliação do perfil de velocidade para o Bocal com Trecho Reto. 


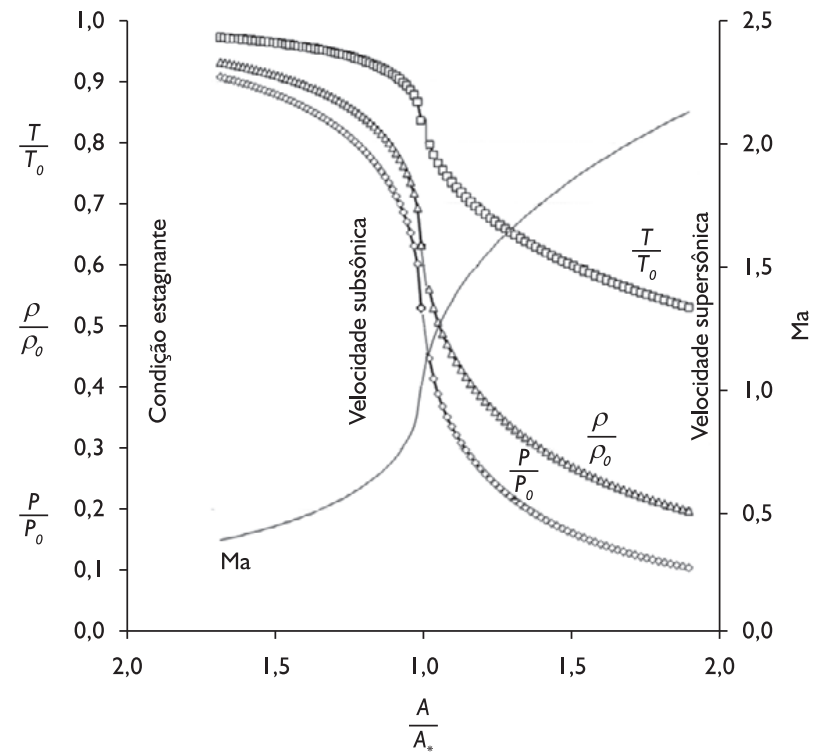

Figura 7. Variação das propriedades do fluxo com a variação $A / A^{*}$ para o Bocal 2.

que $\circ$ trecho reto provoca uma restrição à passagem do gás e queda de eficiência na transição das propriedades. Mesmo que a velocidade Mach seja a mesma, pois é determinada pela pressão do reservatório e pelo diâmetro crítico, o trecho reto provoca uma queda na pressão de saída. A queda na pressão de saída afeta a penetração do jato no banho líquido, potencialmente reduzindo a taxa de descarburação e a eficiência do oxigênio, significando aumento de tempo de sopro e no consumo de oxigênio, no processo produtivo.

A razão associada para existência do trecho reto, em grande parte dos bocais de lanças utilizados na siderúrgica mundial, é o processo de fabricação dos bocais. Porém, os modernos equipamentos CNC (Controle Numérico Computadorizado) permitem a usinagem de geometrias complexas, por exemplo, parabolóides, hiperbolóides, com rigor e precisão dimensional. Pela simulação foi realizado um estudo comparativo com novas propostas de geometria, bocal suavizado 2 e bocal suavizado 3 , da Figura 4, e apresentados na Figura 8, considerando o modelo SST de turbulência.

$\mathrm{Na}$ Figura 8, os bocais suavizados 2 e 3 apresentam uma zona do transônica bem definida, assim como a formação clara das ondas de expansão do fluido. Segundo Ferri, ${ }^{(5)}$ para o correto desenho de um bocal, deve ser usado o método das características para uma condição uniforme de distribuição de velocidade na saída, evitando a formação de ondas compressão ou expansão. As propostas dos bocais suavizados foram feitas de forma empírica, pois o método das características ainda está em estudo pelos autores. Os resultados do modelo de turbulência SST para todas as geometrias simuladas são apresentados na Figura 9, que mostram a ocorrência de expansões supersônicas nos jatos nas saídas dos bocais. O comportamento ideal seria o estado de completamente expandido sem a formação de ondas de expansão. Fazem parte da estrutura de jatos supersônicos zonas de expansão e compressão, e o objetivo de

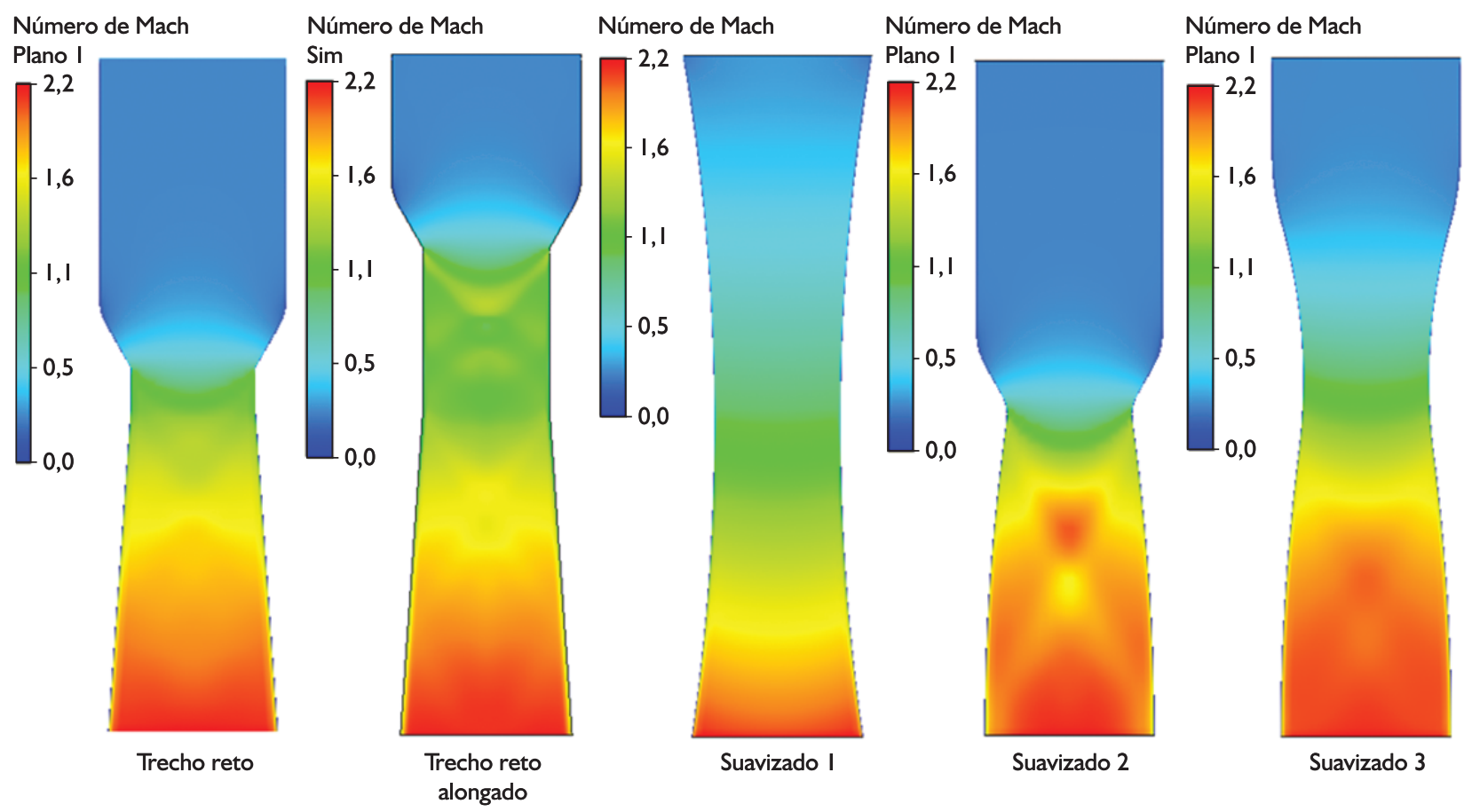

Figura 8. Simulação do modelo de turbulência SST das geometrias propostas: a) Bocal I com trecho reto, b) Bocal com trecho reto alongado, c) Bocal suavizado I, d) Bocal suavizado 2 e e) Bocal suavizado 3. 

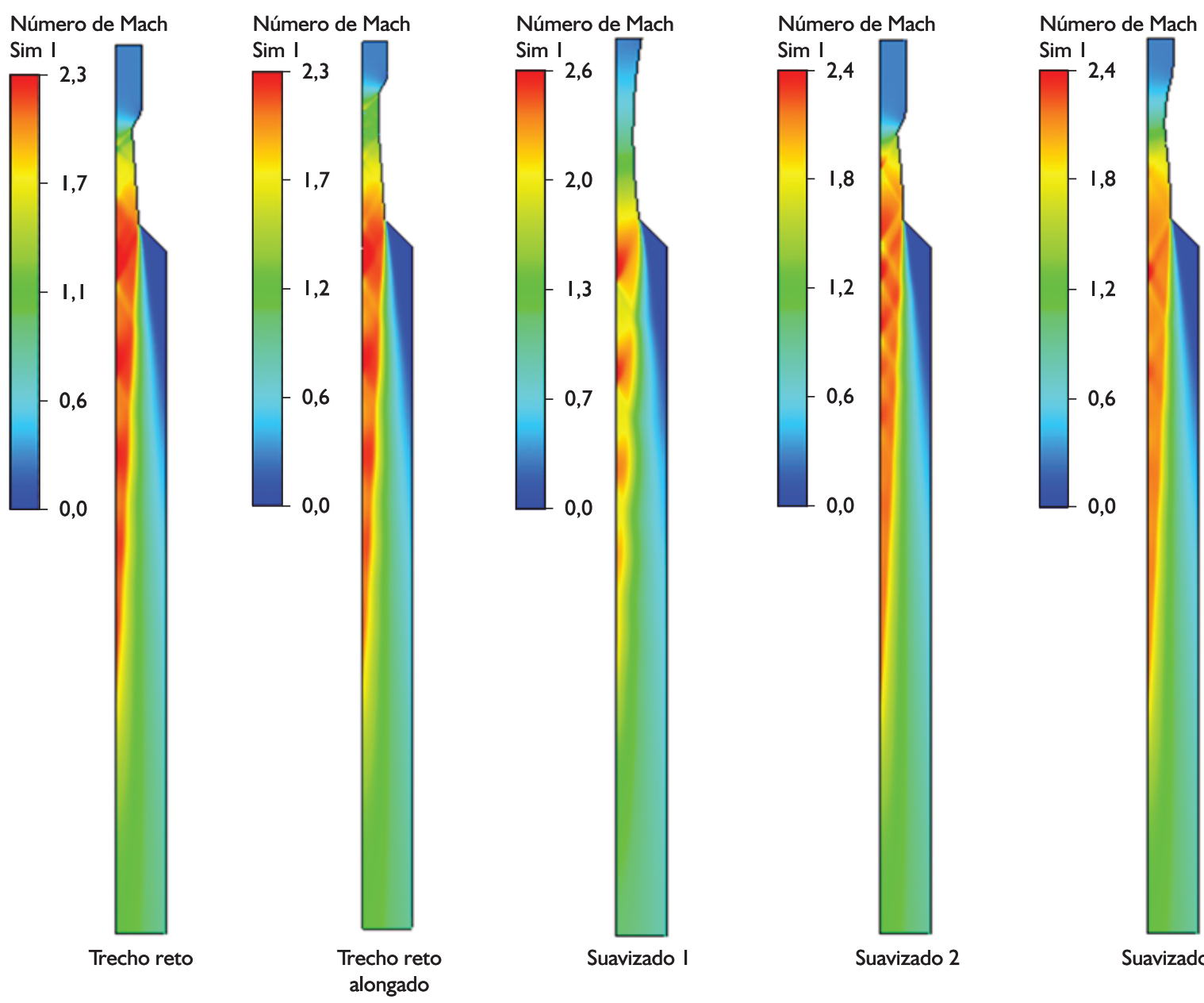

Figura 9. Comparação do núcleo supersônico para as geometrias de bocais testadas.

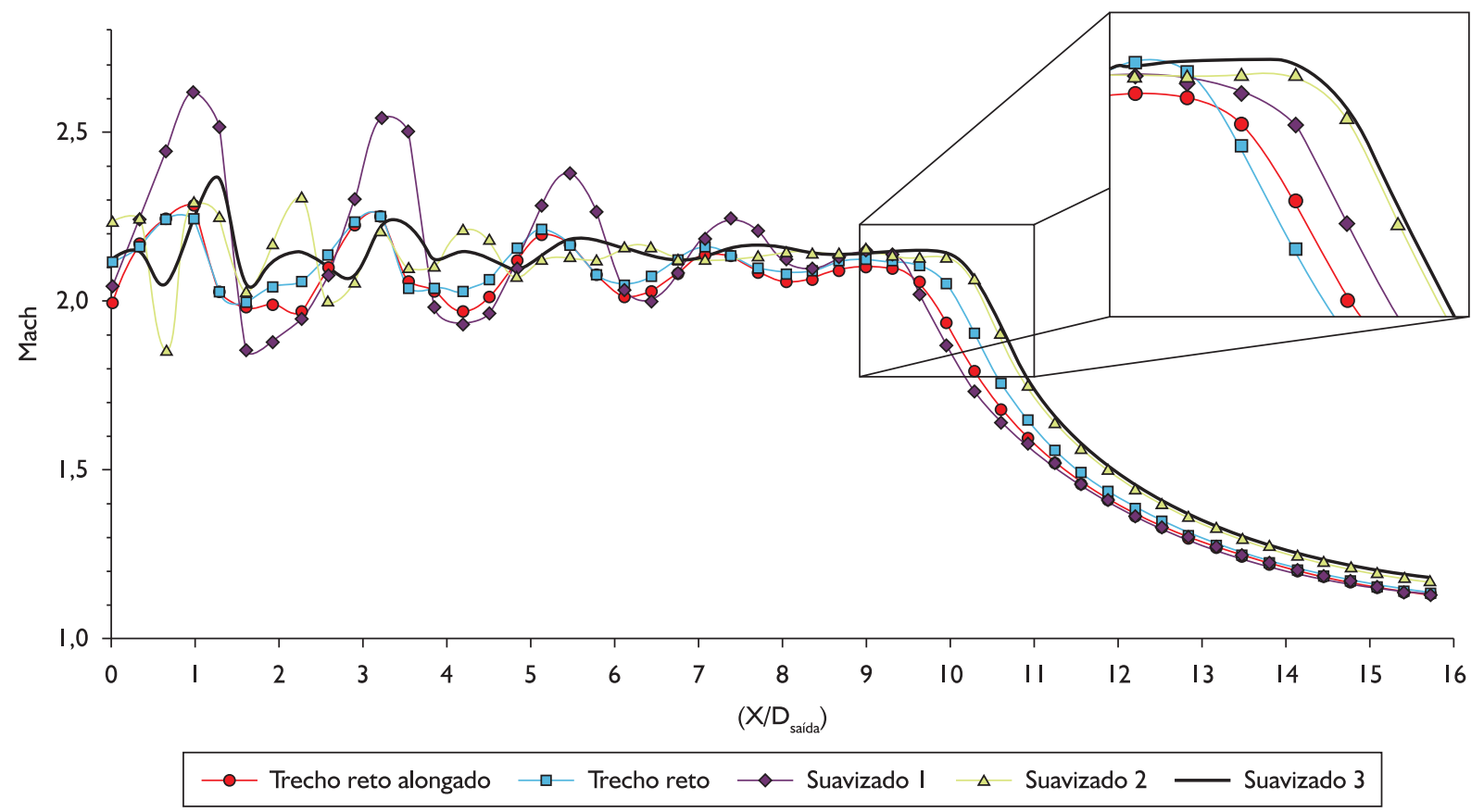

Figura 10. Determinação do comprimento do Núcleo Potencial ao longo do eixo do jato. 
um correto dimensionamento do bocal é minimizá-las. A geometria que melhor atende a este propósito é o bocal suavizado 3 , atingindo o maior comprimento do núcleo potencial. Este comprimento é definido como sendo a região na qual a velocidade no eixo do jato é superior a Mach $=2$. Notadamente no bocal suavizado I, também é possível verificar a influência da geometria, nas ondas de expansão, e as bruscas variações nas propriedades do jato e a interferência da atmosfera no comportamento do jato, em razão do ângulo de saída ser calculado por uma função parabólica. Para uma correta análise dos resultados, é gerado um gráfico com os valores do número de Mach na linha de centro do bocal em relação à distancia da saída. Por meio dessas medidas é possível quantificar com precisão o comprimento do núcleo supersônico e avaliar, entre as geometrias propostas, o melhor resultado para o processo siderúrgico apresentado na Figura 10.

Nota-se que o comprimento do núcleo potencial está em torno de 10 vezes o diâmetro de saída, observando-se que o bocal suavizado 3 apresenta o maior comprimento de núcleo potencial, seguido do bocal suavizado 2 . Os resultados são pertinentes aos encontrados por Alam et al., ${ }^{(6,7)}$ que citam o modelo empírico representado pela Equação 6:

$$
\begin{aligned}
& \frac{\mathrm{I}}{2 \ln \left(I-\mathrm{V} / \mathrm{V}_{\text {SAíA }}\right)}= \\
& 0,6035 \sqrt{\frac{\rho_{\text {Ambiente. }}}{\rho_{\text {Saída }}}} \frac{x}{D_{\text {Saída }}}-0,084 I
\end{aligned}
$$

onde: "V" - velocidade ao longo do eixo, " $\mathrm{V}_{\text {Sáda }}$ " - velocidade na saída do bocal, " $\rho_{\text {Ambiente }}$ " - massa específica ambiente, " $\rho_{\text {Saida }}$ " - massa específica na saída do bocal e " $D_{\text {Saída }}$ " - diâmetro da saída do bocal.

Os bocais trecho reto e suavizado I apresentam as maiores ondas de expansão na saída em comparação com os bocais suavizados 2 e 3 . Esse comportamento reduz em aproximadamente $1 \%$ o comprimento núcleo potencial e menores números de Mach para uma mesma distância após o núcleo potencial.

\section{CONCLUSÃO}

As principais conclusões do trabalho são:

Não é necessário o trecho reto na região da garganta dos bocais supersônicos, pois é prejudicial à transição das propriedades do gás;

A queda de eficiência provoca perdas prematuras de velocidade do jato e, consequentemente, perda de penetração e descarburação do banho;

É necessário um maior aprofundamento no método das características para aprimoramento das geometrias de bocais para sopros de oxigênio;

As indústrias siderúrgicas já têm ao seu alcance a possibilidade de obter geometrias de bocais eficientes, reduzindo o tempo de sopro.

\section{Agradecimentos}

Os autores do trabalho agradecem à Universidade Federal de Minas Gerias - UFMG pela cessão dos laboratórios, e ao Prof. Ronaldo Sampaio, da RS Consultants, pelo apoio para apresentação dos trabalhos.

\section{REFERÊNCIAS}

I GLASS, D. R.; SMITH, G. C. Nozzle theory. In: GAINES, J. M. (Ed.). BOF steelmaking. New York: The Iron and Steel Society of the American Institute of Mining, Metallurgical and Petroleum Engineers, 1976. v. 3, Cap.8.

2 SHAPIRO, H. N.; MORAM, M. J. Fundamentals of engineering thermodynamics. ed. New York: John Wiley \& Sons, 1988. p. 4I7-35.

3 NASCIMENTO, L. M. Estudo do efeito da pressão e da geometria de bico de lança no comportamento supersônico do oxigênio através de análise por CFD. 20I0. (Trabalho de Final de Curso) - Escola de Engenharia da Universidade Federal de Minas Gerais, Belo Horizonte, 2010.

4 ANSYS. Innovative Turbulence Modeling: SST Model in ANSYS CFX. Technical brief ansys. Disponível em: <http:// www.ansys.com >. Acesso em: 29 dec. 2006.

5 FERRI, A. Elements of aerodynamics of supersonic flows. New York: The MacMillan, 1949.

6 ALAM, M. et al. Computational fluid dynamics modeling of supersonic coherent jets for electric arc furnace steelmaking process. Metallurgical and Materials Transactions B, v. 4I, n. 6, p.1354-67, Dec. 2010. http://dx.doi. org/I0.1007/s I |663-0I0-9436-7

7 ALAM, M. et al. Computational fluid dynamics simulation of supersonic oxygen jet behavior at steelmaking temperature. Metallurgical and Materials Transactions B, v. 4I, n. 3, p. 636-45. Jun. 20I0. http://dx.doi.org/I0.1007/ sl|663-010-934|-0

Recebido em: 17/09/20II

Aceito em: 13/02/2012 